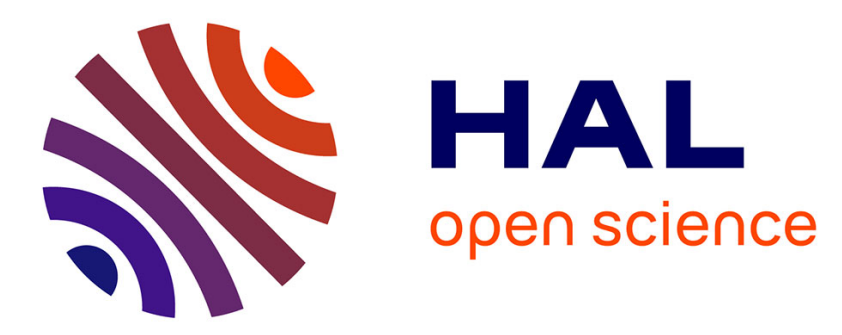

\title{
Spray-assisted alignment of Layer-by-Layer assembled silver nanowires: a general approach for the preparation of highly anisotropic nano-composite films
}

H. Hu, M. Pauly, O. Felix, G. Decher

\section{- To cite this version:}

H. Hu, M. Pauly, O. Felix, G. Decher. Spray-assisted alignment of Layer-by-Layer assembled silver nanowires: a general approach for the preparation of highly anisotropic nano-composite films. Nanoscale, 2017, 9 (3), pp.1307-1314. 10.1039/c6nr08045f . hal-03562516

\author{
HAL Id: hal-03562516 \\ https://hal.science/hal-03562516
}

Submitted on 9 Feb 2022

HAL is a multi-disciplinary open access archive for the deposit and dissemination of scientific research documents, whether they are published or not. The documents may come from teaching and research institutions in France or abroad, or from public or private research centers.
L'archive ouverte pluridisciplinaire HAL, est destinée au dépôt et à la diffusion de documents scientifiques de niveau recherche, publiés ou non, émanant des établissements d'enseignement et de recherche français ou étrangers, des laboratoires publics ou privés. 


\title{
Spray-assisted alignment of Layer-by-Layer assembled silver nanowires: a general approach for the preparation of highly anisotropic nano-composite films
}

\author{
H. Hu, ${ }^{a}$ M. Pauly, ${ }^{* a, b}$ O. Felix ${ }^{a}$ and G. Decher ${ }^{* a, b, c, d}$ \\ anstitut Charles Sadron, CNRS (UPR22), 23 rue du Loess, F-67034 Strasbourg, France. \\ bFaculté de Chimie, Université de Strasbourg, 1 rue Blaise Pascal, F-67008 Strasbourg, France \\ International Center for Frontier Research in Chemistry, 8 allée Gaspard Monge, F-67083 \\ Strasbourg, France \\ 'Excellence Cluster "Nanostructures in Interaction with their Environment" (LabEx NIE), 23 rue \\ du Loess, F-67034 Strasbourg, France
}

\section{Corresponding author:}

E-mail: matthias.pauly@ics-cnrs.unistra.fr, decher@unistra.fr

\begin{abstract}
The present article focuses on the build-up and the properties of oriented silver nanowire monolayer films and Layer-by-Layer assembled multilayer films. We describe the templatefree oriented spray-assisted assembly of silver nanowires at solid/air-interfaces using Grazing Incidence Spraying, a simple and versatile approach that allows the formation of highly oriented thin films with a tunable density and in-plane orientation. Depending on the spraying conditions the nematic order parameter, which describes the angular spread of misaligned nanowires, can be as high as 0.98 (a value of 1.00 corresponding to a perfectly parallel alignment). The combination with the Layer-by-Layer assembly allows building multilayer thin films possessing in-plane anisotropy. In order to demonstrate that the local alignment does not cancel out on the macroscopic scale but leads to direction-dependent properties, we use linearly polarized UV-Vis-NIR spectroscopy to probe the selective excitation of the transverse and longitudinal localized plasmon resonances of the nanowires. The polarization efficiency of the thin films increases strongly with the in-plane density, the degree of orientation, and the number of silver nanowire layers. Multilayer films containing 4 layers of nanowires oriented in the same direction reach a polarization efficiency of up to $97 \%$ in the near-infrared region.
\end{abstract}

\section{Introduction}

The development of nanocomposite materials of high compositional and structural complexity, for example bio-inspired materials, is generally thought to be very important but not much explored due to the lack of appropriate methods for their preparation. ${ }^{1}$ Interesting materials properties arise either from the composition of a material (i.e. the number and the chemical nature of its constituents) and/or from the complexity of its structure. ${ }^{2}$ However, the number of different constituents in man-made materials is presently limited and their hierarchical organization is often very simple. With respect to the preparation of multimaterial thin films with a high level of control over the spatial positioning of their constituents, Layerby-Layer (LbL) assembly has gained its merits as a simple and highly versatile nanofabrication method. ${ }^{3,4}$ Multifunctional coatings with unique biomedical, electrical, optical, mechanical and magnetic properties can routinely be prepared by LbLassembly ${ }^{4,5}$ as 
it allows the combination of a large variety of components such as polyelectrolytes, spherical nanoparticles, ${ }^{6}$ nanorods ${ }^{7}$ and nanowires ${ }^{8}$ in a single LbL-film. While the sequence of components in layered multi-material films can be very well controlled by LbL-assembly, tuning of the in-plane anisotropy has not yet been achieved. This has been a severe drawback since one-dimensional (1D-) nanomaterials, such as nanotubes, nanorods and nanowires, have been receiving increasing attention in the last few years because of their anisotropic physical properties and their potential applications in opto-electronic nanodevices. ${ }^{9-11}$

Among the broad range of inorganic 1D-nanomaterials, silver nanowires (AgNWs) are one of the most investigated ones ${ }^{10,12}$ as they could find applications as transparent conductive electrode materials, ${ }^{13}$ plasmonic waveguides, ${ }^{9,14}$ antennas ${ }^{15}$ or sensors. ${ }^{16,17}$

A variety of methods, ${ }^{18}$ including Langmuir-Blodgett deposition, ${ }^{19,20}$ blown bubble film transfer, ${ }^{21}$ dip-coating, ${ }^{22} \mathrm{H}$-bar dip-coating, ${ }^{23}$ doctor-blade casting, ${ }^{24}$ capillary printing ${ }^{25}$ and the use of electrical or magnetic fields, ${ }^{26}$ have been suggested to control the assembly and inplane alignment of rod-like nano-objects. Other techniques use flow-induced shear in fluidic cells, ${ }^{27}$ in a capillary, ${ }^{17,28}$ or by evaporating spray droplets arriving on hot surfaces. ${ }^{29}$ However, these methods are limited in their ability to yield uniformly aligned films over large areas, they are restricted to small groups of components or they fail to produce compositional control along the z-direction.

Recently, we have introduced "Grazing Incidence Spraying" (GIS) as a new method for the preparation of oriented monoand multilayer films of cellulose microfibrils ${ }^{30}$ or of monolayers of metallic nanowires and nanorods. ${ }^{31}$ While it is somewhat related to "blow-alignment", ${ }^{32}$ or to "combing", 33 GIS is based on spray-assisted shear-induced assembly and can be combined with the LbL-approach in order to extend it toward the preparation of in-plane anisotropic multilayer films.

In a previous study, ${ }^{31}$ we have shown that oriented monolayer thin films of 1D-nanoparticles such as gold nanorods and AgNWs can be efficiently oriented using GIS. We have also shown that the quality of alignment increases with the length of the deposited nano-objects. Here we use GIS for the controlled alignment of AgNWs in mono- and multilayer architectures for preparing well-aligned thin films of controlled thickness homogeneous over large areas. In order to demonstrate that the local topological orientation of 1D-nano-objects induces macroscopic anisotropic properties, we use linearly polarized UV-Vis-NIR spectroscopy to probe the selective excitation of the transverse and longitudinal localized plasmon resonances of the nanowires. The anisotropic character of the physical properties (i.e. the polarization efficiency) of these mono- and multilayer architectures strongly increases with the in-plane density, the degree of orientation, and the number of AgNW layers.

\section{Results and discussion}

\section{Monolayers of oriented silver nanowires}

AgNWs were synthesized by a polyol reduction procedure as previously reported in the literature (for a detailed description, see the ESI + ), ${ }^{17,34}$ which results in nanowires that have a cross section of $47 \pm 6 \mathrm{~nm}$ and a length of $4.2 \pm 1.5 \mu \mathrm{m}$, as determined by Transmission Electron Microscopy (TEM) (Fig. S1†).

The oriented deposition of AgNWs on different surfaces was carried out by spraying the nanowire suspension at an angle of $15^{\circ}$ onto a receiving surface using the GIS approach (Fig. 1a), which results in a dense monolayer of aligned AgNWs (Fig. 1b). AgNW monolayers were prepared on glass slides or on silicon wafers, both of which were previously coated with a layer of poly(ethyleneimine) (PEI). 
The distribution of the angle $\theta$ between each nanowire principal axis and the spraying direction is extracted from the Scanning Electron Microscopy (SEM) pictures using the plugin Orientation ${ }^{35}$ developed for ImageJ, ${ }^{36}$ which is based on the analysis of the structure tensor in a local neighbourhood (Fig. 1C-e and S2+). A 2D nematic order parameter $S$ can be calculated from this angular distribution

$$
S_{2 D}=\left\langle 2 \cos ^{2} \theta-1\right\rangle
$$

where the chevrons denote the weighted average over the distribution of $\theta, S=0$ corresponds to a random distribution (isotropic films, i.e. $\theta$ is equally distributed between $90^{\circ}$ and $-90^{\circ}$ ) and $S=1$ corresponds to a perfectly parallel orientation (fully anisotropic films, i.e. $\theta=0^{\circ}$ for all AgNWs). The highest ordering of AgNWs (Fig. 1) was obtained at $1 \mathrm{~cm}$ downstream from the impact point of the spray jet on the substrate $(S=0.98$, Fig. $1 \mathrm{~b}$ and $\mathrm{c})$.
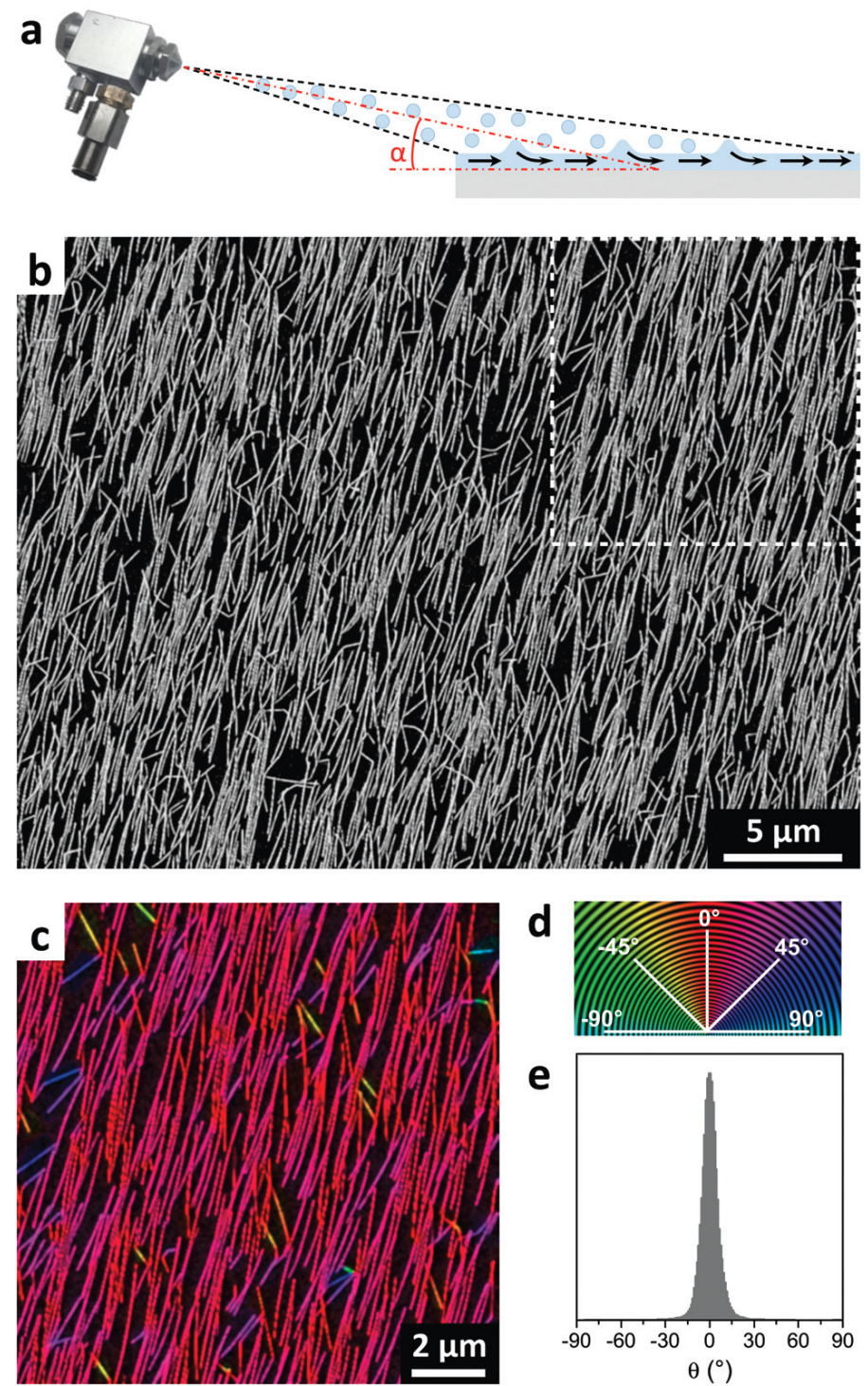

Fig. 1 (a) Schematic representation of the GIS deposition technique. (b) SEM picture of an AgNW monolayer deposited on PEI-coated silicon wafer. (c) Orientation analysis of the dotted square portion of panel (b), in which the nanowires are colored according to their orientation. (d) Color-code used for the orientation analysis. (e) Distribution of the nanowire angle $\theta$ relative to the spraying direction. 
This corresponds to $\sim 97 \%$ of the nanowires aligned within $\pm 15^{\circ}$ of the spraying direction, and to $\sim 66 \%$ of the nanowires aligned within $\pm 5^{\circ}$. The degree of orientation strongly depends on the distance away from the impact point along the flow direction. For instance, $\mathrm{S}$ is reduced to 0.87 at $1.5 \mathrm{~cm}$ away from the spray jet impact point. Generally speaking, $S$ was above 0.80 for all the samples made with AgNWs following the method used here. We believe that the spraying at small angles produces a thin liquid film on the receiving surface whose flow across the surface in the direction away from the nozzle exercises sufficient shear for orienting the 1D-nanomaterials during their adsorption fromthe suspension. The nanowire density in the monolayer, and thus the in-plane plasmonic coupling ${ }^{37}$ between different $\mathrm{AgNWs}$ in the same layer is conveniently tuned by varying the spraying time (Fig. 2).
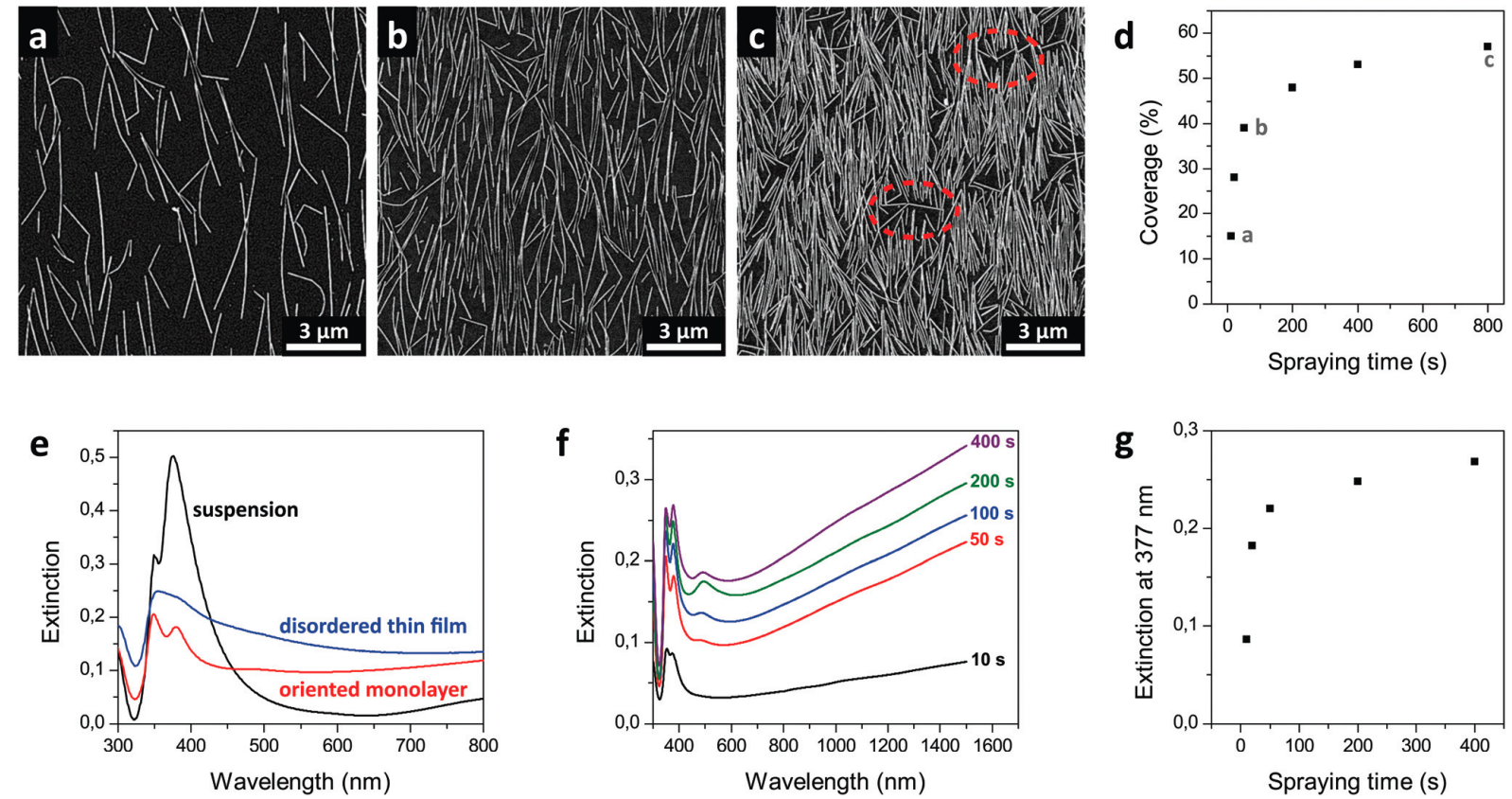

Fig. 2 (a-d) SEM pictures of AgNW monolayers deposited for $10 \mathrm{~s}(\mathrm{a}), 50 \mathrm{~s}$ (b) and $800 \mathrm{~s}(\mathrm{c})$, and surface coverage measured on SEM pictures as a function of the spraying time (d). The circled areas in panel (c) highlight regions in which the nanowire density is lower around misaligned nanowires. (e) UV-Vis-NIR extinction spectra of the nanowire suspension, a disordered AgNW thin film and an oriented AgNW monolayer thin film. (f) UV-Vis- NIR extinction spectra of oriented monolayer thin films deposited for $10 \mathrm{~s}, 50 \mathrm{~s}, 100 \mathrm{~s}, 200 \mathrm{~s}$ and $400 \mathrm{~s}$, and (g) extinction at $377 \mathrm{~nm}$ as a function of spraying time.

The surface coverage was varied between $15 \%$ for 10 seconds spraying, and up to $60 \%$ when the spraying is maintained for a few minutes (Fig. 2d). AgNWs only adsorb in areas where a PEl-coated interface is available (Fig. 2a-c), and do not form an adlayer on top of already adsorbed AgNWs. In competitive random sequential adsorption, a jamming coverage will be reached that depends on the size and shape of the adsorbing species and at which the surface coverage reaches a plateau. This adsorption mechanism also explains why the density is lower around misaligned nanowires or deformed nanowires (see for instance the circled areas in Fig. 2c). The extinction spectrum of AgNWs displays two peaks in the UV-Vis region and a broad band extending in the near-infrared (NIR) region due to the localized surface plasmon resonances (LSPR). For AgNWs in suspension (Fig. 2e), two peaks at 349 and $377 \mathrm{~nm}$ are observed. Kottman et al. have observed that silver nanowires with a non-circular cross-section display multiple transverse resonance peaks. ${ }^{38}$ These peaks are commonly assigned to the outof-plane quadrupole resonance and the out-of-plane dipole resonance of nanowires respectively, ${ }^{20,39}$ although this assignment has, to the best of our knowledge, not yet been 
confirmed by simulations. A broad band starting at $\sim 650 \mathrm{~nm}$ and extending towards the near infrared region can be assigned to the longitudinal mode of the LSPR. UV-Vis extinction with unpolarized light has also been measured for a disordered sample obtained by dropcasting and an oriented monolayer (Fig. 2e). The transverse LSPR peaks are significantly broadened and slightly redshifted due to plasmonic coupling between neighbouring AgNWs. The total extinction increases with the spraying time as the AgNW density increases in the monolayer (Fig. 2f), in particular the extinction measured at $378 \mathrm{~nm}$ (i.e. at a transverse LSPR peak, Fig. $2 \mathrm{~g}$ ). Furthermore, a new peak at $\sim 450 \mathrm{~nm}$ appears for spraying times above 50 seconds. The intensity of this peak increases for longer spraying times (i.e. for a higher AgNW density on the surface), and it can be assigned to a new mode arising from the coupling of dipolar transverse LSPR modes of individual nanowires. ${ }^{37}$
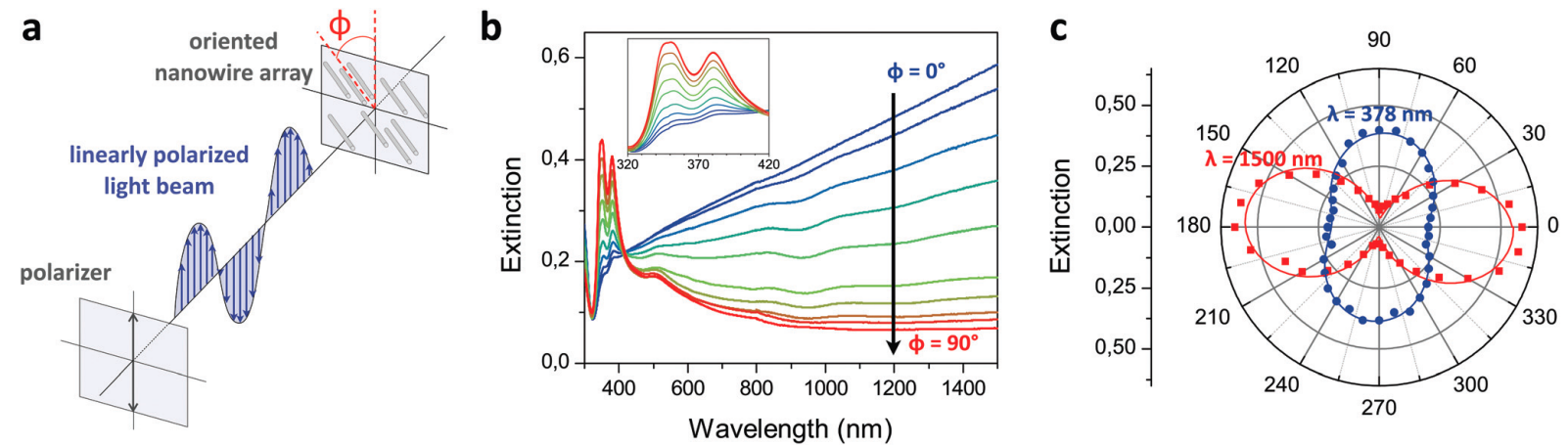

Fig. 3 (a) Scheme describing the polarized UV-Vis-NIR extinction measurement, in particular the angle $\phi$ between the light polarization plane and the nanowire orientation direction. (b) UV-Vis-NIR extinction spectra of an oriented nanowire monolayer thin film measured for various polarization angles $\phi$. (c) Polar plot of the extinction at $378 \mathrm{~nm}$ (transverse LSPR) and $1500 \mathrm{~nm}$ (longitudinal LSPR) of an oriented AgNW monolayer thin film as a function of the polarizer angle $\phi$.

Polarized UV-Vis-NIR spectra of oriented AgNW arrays are used to evidence the macroscopic anisotropic character of the optical properties due to the local anisotropy. Indeed, both the longitudinal and the transverse modes of the localized surface plasmon resonance are independent of the polarization of the incident light in the non-oriented film (Fig. $\mathrm{S3}^{+}$). When the nanowires are aligned, strong polarization effects are observed with respect to the polarization directions of the longitudinal and transverse modes (Fig. 3). The polarized extinction was measured as a function of the angle $\phi$ between the incident light beam polarization plane and the orientation direction (Fig. 3a and b). For $\phi=0^{\circ}$ (i.e. polarization parallel to the long axis of the nanowires), the longitudinal LSPR mode is excited as seen from the high extinction in the NIR. In contrast, for $\phi=90^{\circ}$ (i.e. polarization perpendicular to the long axis of the nanowires), the transverse LSPR modes are excited resulting in a high extinction in the UV-visible region and in the appearance of a small peak at $450 \mathrm{~nm}$ related to the coupling of transverse LSPR modes of individual nanowires. ${ }^{37}$ Consequently, for intermediate polarization angles both modes are excited. The extinctions at $378 \mathrm{~nm}$ and 1500 $\mathrm{nm}$ are depicted in Fig. $3 \mathrm{c}$ in a polar plot as a function of the polarization angle $\phi$. Fig. $3 \mathrm{~b}$ and c illustrate nicely that oriented nanowire films are wavelength-dependent polarizers, as light is polarized in perpendicular directions below and above $425 \mathrm{~nm}$. Another consequence is the high contrast of the reflected intensity of oriented nanowire thin films under crosspolarizers, demonstrating the macroscopic homogeneity of the optical properties (see Fig. S4†). 


\section{Multilayers of oriented silver nanowires}

As the orientation process has been demonstrated to be efficient for the build-up of an aligned AgNW monolayer, we used the Layer-by-Layer approach in order to fabricate multilayer films. $\mathrm{PEI}$, poly(sodium 4-styrenesulfonate) (PSS) and poly(allylamine hydrochloride) (PAH) were used as spacer layers between the nanowire layers, and the architecture of multilayer samples can be denoted as PEI/AgNW/[PEI(PSS/PAH)5PSS/PEI/AgNW] $]_{n-1}, \mathrm{n}$ indicating the number of individual nanowire layers.
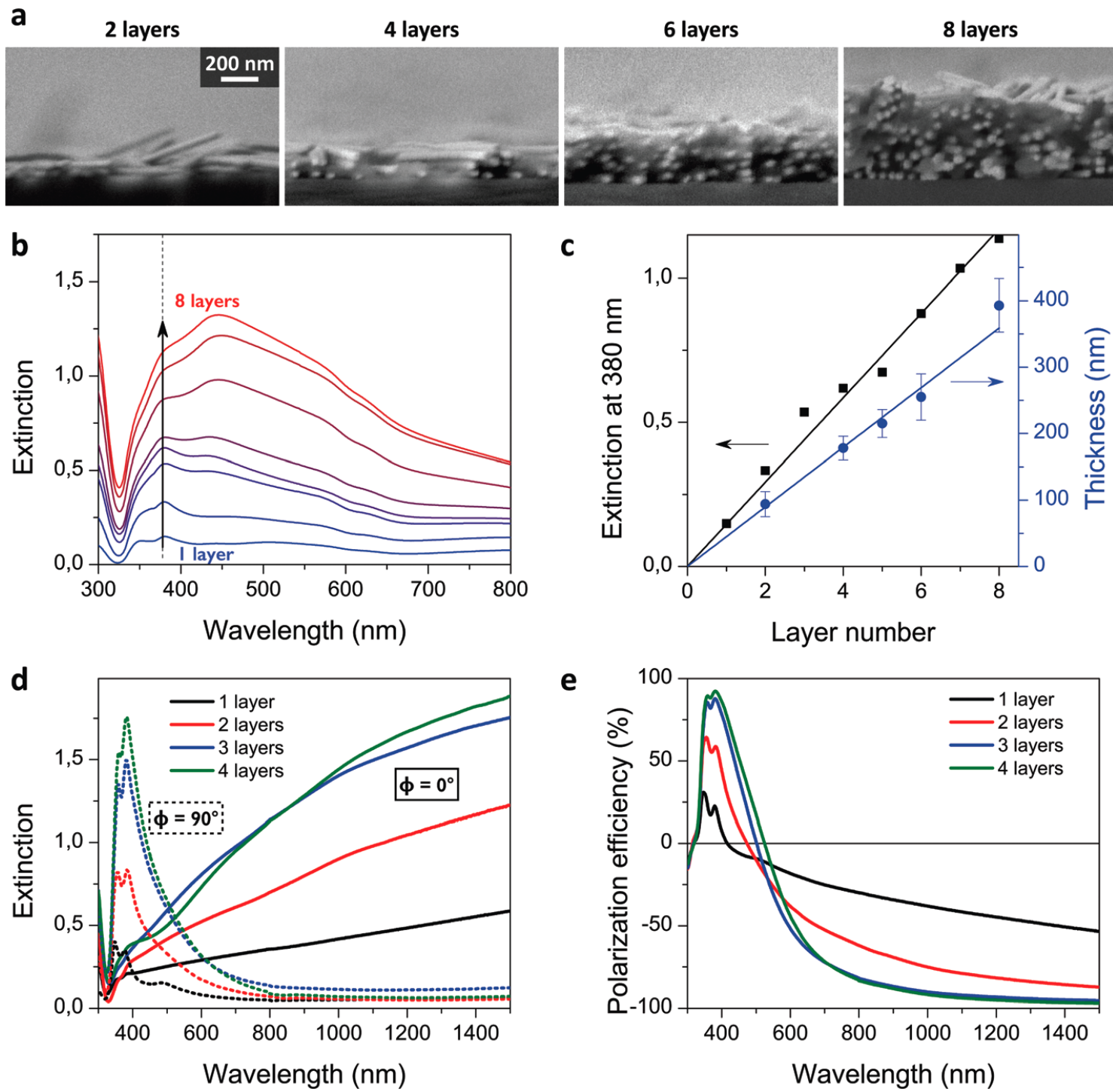

Fig. 4 (a) Cross-section SEM pictures of oriented thin films composed of $n=2,4,6$ and 8 AgNW layers. (b) Unpolarized extinction spectra of thin films of different thicknesses. (c) Extinction at $380 \mathrm{~nm}$ and thickness measured from the cross-section SEM pictures as a function of silver nanowire layer number $n$. (d) Extinction spectra for light polarized parallel (full lines) and perpendicular (dashed lines) to the orientation direction for various layer numbers $n$. (e) Polarization efficiency for various layer numbers $n$ (see eqn (2)).

The multilayer build-up was monitored by cross-section SEM (Fig. 4a) and by determining the extinction (Fig. 4b) as a function of the number of deposited layers. Note that the term "AgNW layer" refers to the sequential adsorption of LbL films, but that such films resemble more a "woodpile"-like structure than a regularly layered stack. However, it appears clearly from the SEM pictures (Fig. 4a) that the film thickness increases proportionally to the number of AgNW 
deposition cycles, with a growth increment of $\sim 50 \mathrm{~nm}$ per repeating unit (Fig. $4 \mathrm{c}$ ), which fits well with the combined thickness of the AgNWs and of the polyelectrolyte spacer layers. This linear growth is corroborated by the increase of the extinction at $380 \mathrm{~nm}$ as the number of deposited AgNW layers increases (Fig. 4c). The two transverse LSPR bands are broadened and strongly overlap, and a new broad extinction band appears at around $450 \mathrm{~nm}$ whose intensity grows as the number of layers increases, which arises from plasmon coupling between neighbouring layers. ${ }^{37}$

Similarly to the oriented monolayer discussed above, the optical response of oriented multilayer thin films is polarization-dependent. Fig. $4 \mathrm{~d}$ displays the extinction of the monolayer and multilayers with $n=2-4$ for $\phi=0^{\circ}$ and $\phi=90^{\circ}$. Similarly to monolayer films, the absorbance in the NIR range is high when the longitudinal LSPR band is excited and the absorbance in the near-UV range is high when the transverse LSPR bands are excited using the appropriate polarization directions in each case. The polarization efficiency (P.E.) is conveniently defined as the normalized difference between the transmittance of light polarized parallel $\left(T_{\|}\right)$and perpendicular $\left(T_{\perp}\right)$ to the direction of nanowire orientation.

$$
P . E .=\frac{T_{\|}-T_{\perp}}{T_{\|}+T_{\perp}}
$$

The polarization efficiency shows a positive maximum at the position of the transverse LSPR bands in the near-UV range, and becomes more and more negative when extending towards the NIR (Fig. 4e). Furthermore, the polarization efficiency increases strongly with the number of deposited AgNW layers. P.E. is equal to $31 \%$ at $347 \mathrm{~nm}$ and $-57 \%$ at $1500 \mathrm{~nm}$ for a single layer of aligned nanowires, and reaches $92 \%$ and $-97 \%$ for the 4 -layer sample in the UV and NIR respectively. Note that P.E. does not increase much when adding a fourth AgNW layer to a multilayer composed of three AgNW layers because P.E. is already getting close to its maximum value of $100 \%$.

\section{Conclusions}

In summary, we have shown that GIS is a very easy and versatile approach for orienting AgNWs. The resulting anisotropic thin films display optical properties that are in turn highly anisotropic. Moreover, multilayer thin films can be fabricated by combining this method with the Layer-by-Layer technique. The degree of orientation is high, with at least $95 \%$ of the nanowires being oriented within $\pm 15^{\circ}$ with respect to the main direction.

It should be highlighted that GIS is a process that is very easy to implement and scale up, as it does not require costly equipment or complicated sample preparation. Once the parameters are optimized, deposition of a layer takes a few tens of seconds, which can probably be reduced to shorter times by increasing the nanowire/substrate interaction. A single static spraying nozzle as used in this study allows covering homogeneously a few $\mathrm{mm}^{2}$, and this can already be scaled-up to several $\mathrm{cm}^{2}$ using a mobile nozzle while the scale-up to $\mathrm{m}^{2}$ is expected to be possible either by using an array of nozzles, or by scanning the nozzle(s) over the substrate. Such homogeneous anisotropic large area coatings are difficult to prepare by other alignment techniques such as Langmuir-Blodgett or doctor-blading.

Finally, the tunability is very high, as GIS can in theory be applied to any 1D-nanomaterial on any type of substrate (oxide, metallic, polymeric, flexible or not, etc.) provided that there is an attractive interaction between the substrate and the deposited nanowires/nanorods (which can be adjusted by playing with substrate/nano-object surface functionalization). The combination with the LbL approach ${ }^{3-5}$ furthermore opens perspectives towards multi- 
material architectures, in which different (nano)materials can be deposited in each layer, having independent control on the composition, density and orientation of each layer. As an example, Fig. S5t shows a 2-layer thin film in which the second AgNW layer is aligned perpendicularly to the first one. One could imagine even more complex architectures, for instance by varying the angle between the layers or the number of layers. The LbL approach is also well known as a technique ideally suited for the fabrication of multi-material composite thin films that can comprise a large variety of materials. ${ }^{40}$ The combination of the tunable inplane anisotropy demonstrated in this paper and the well-established control of the layer sequence (i.e. anisotropy in the direction of the layer normal) offered by LbL assembly allows envisioning multi-material nanoscale functional thin films that would enable new applications for example in optoelectronics, (bio)chemical sensing or for transparent conductive electrodes and solar cells.

\section{Materials and methods}

\section{Chemicals}

Poly(ethyleneimine) (PEI, $\overline{M_{n}} \approx 60000 \mathrm{~g} \cdot \mathrm{mol}^{-1}$ ), poly(sodium 4-styrenesulfonate) (PSS, $\overline{M_{w}} \approx$ $70000 \mathrm{~g} \cdot \mathrm{mol}^{-1}$ ), poly(allylamine hydrochloride) (PAH, $\overline{M_{w}} \approx 15000 \mathrm{~g} \cdot \mathrm{mol}-1$ ), poly(vinylpyrrolidone) (PVP, $\overline{M_{w}} \approx 40000 \mathrm{~g} \cdot \mathrm{mol}-1$ ), silver nitrate and glycerol were purchased from Sigma-Aldrich (Saint Quentin Fallavier, France). Sodium chloride was purchased from Carl Roth $\mathrm{GmbH}$ (Karlsruhe, Germany). All the chemicals were used without further purification. The aqueous solutions were prepared with ultrapure water (resistivity $=18.2$ $\mathrm{M} \Omega \cdot \mathrm{cm}$, Milli-Q Gradient system, Millipore, Molsheim, France).

$\mathrm{PEI}$ solutions were freshly prepared by direct dissolution of $2.5 \mathrm{mg} \cdot \mathrm{mL}-1$ of the polymer in ultrapure water. PSS solutions were prepared in $\mathrm{NaCl}$ solution $(0.5 \mathrm{M})$ at a concentration of $0.62 \mathrm{mg} \cdot \mathrm{mL}-1$. PAH solutions were prepared in $\mathrm{NaCl}$ solution $(0.5 \mathrm{M})$ at a concentration of $0.29 \mathrm{mg} \cdot \mathrm{mL}-1$.

\section{Synthesis of Ag nanowires}

Silver nanowires were synthesized by a polyol reduction procedure previously reported in the literature (for detailed description, see the ESI + ). ${ }^{17,34}$ Briefly, a glycerol solution of $\mathrm{AgNO}_{3}, \mathrm{NaCl}$ and PVP is heated from room temperature to $210^{\circ} \mathrm{C}$, which leads to Ag+ reduction and to the formation of silver nanowires that typically possess a pentagonal cross-section. The wires, coated in situ with PVP, form a stable suspension in water. The synthesis as performed in our laboratory results in nanowires which have a cross section of $47 \pm 6 \mathrm{~nm}$ and a length of $4.2 \pm$ $1.5 \mu \mathrm{m}$, as determined by Transmission Electron Microscopy (TEM) (see Fig. S-1†).

\section{Oriented deposition of silver nanowire layers}

An aqueous suspension of silver nanowires $\left(0.12 \mathrm{mg} \cdot \mathrm{mL}^{-1}\right)$ was sprayed on PEl-coated substrates (either glass slides or silicon wafers) using a home-made spraying system (see the $\mathrm{ESI}+$ ) equipped with 2-fluid nozzles (internal diameter: $300 \mu \mathrm{m}$, Spraying Systems, Wheaton, IL) for 200 seconds unless otherwise specified. The angle between the central axis of the cone of the spray jet and the receiving substrate was fixed at $\alpha=15^{\circ}$ (Fig. 1a), and the distance between the nozzle and the substrate was held at a constant distance of $1 \mathrm{~cm}$. The liquid flow

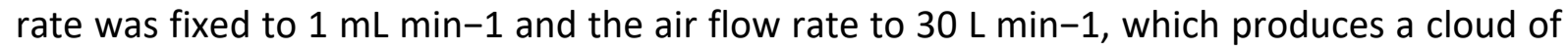
droplets with a diameter in the range of 5-20 $\mu \mathrm{m}$ as determined by Phase Doppler Interferometry (PDI, data not shown here). Following deposition, the thin film was rinsed with Milli-Q water and dried using air flow. 
For silver nanowire multilayer build-up, a polyelectrolyte multilayer spacer was deposited between each silver nanowire layer using air-pump spray cans (Roth, Lauterbourg, France).

Each polyelectrolyte solution was sprayed for 10 seconds, followed by a 10 seconds rinsing step. Layer pairs of PSS and PAH were selected as spacer layers between the nanowire layers. The polyelectrolyte deposition sequence thus consisted of 1 layer of $\mathrm{PEI}$, followed by 5 alternate layers of PSS and PAH, followed by another layer of PSS and finally a layer of PEI. The subsequent silver nanowire layer was sprayed under the same conditions as those used for the first layer, and the whole process can be repeated several times. The architecture of all multilayer samples discussed here can therefore be denoted as $\mathrm{PEI} / \mathrm{AgNW} /\left[\mathrm{PEI}(\mathrm{PSS} / \mathrm{PAH}) 5 \mathrm{PSS} / \mathrm{PEI} / \mathrm{AgNW}_{\mathrm{n}-1}, \mathrm{n}\right.$ indicating the number of individual nanowire layers.

\section{Characterization}

The nanowire size distribution was determined from the images obtained with a Tecnai G2 FEI Transmission Electron Microscope (TEM). Thin films were imaged by Scanning Electron Microscopy (SEM) using a JEOL 6700F microscope equipped with a field emission gun (FEG) and operating at $3 \mathrm{kV}$. The image analysis was performed using ImageJ. ${ }^{36}$ The orientation analysis was done with OrientationJ, ${ }^{35}$ a plugin developed for ImageJ, which is based on the analysis of the structure tensor in a local neighbourhood (see Fig. S-2 + for a detailed description of the orientation analysis procedure). UV-Visible-Near Infrared spectroscopy was carried out with a Cary 5000 spectrometer (Agilent) in dual beam configuration. The extinction for the thin film samples was measured through a circular aperture with $5 \mathrm{~mm}$ diameter and a blank substrate was used as a reference. A Glan-Taylor polarizer has been used for polarized UV-Vis-NIR spectroscopy.

\section{Acknowledgements}

The authors thank Marc Schmutz and the Electron Microscopy Platform (ICS, France) for TEM measurements, Cedric Leuvrey (IPCMS, France) for SEM measurements, and Martin Brinkmann (ICS, France) for giving access to his optical microscope. M.P. thanks the "Chaire d'Excellence" program of CNRS and the Université de Strasbourg for partial funding. G.D. thanks the Institut Universitaire de France for financial support.

\section{†Electronic supplementary information (ESI) available:}

Detailed description of the experimental procedure, TEM pictures of the silver nanowires and their size distribution, description of the electron microscopy image analysis procedure, polarized optical properties of a non-oriented thin film, optical micrographs between crossedpolarizers of the oriented silver nanowire layers, and the SEM picture of a thin film composed of two AgNW layers with different orientation directions. See DOI: $10.1039 / \mathrm{c} 6 \mathrm{nr} 08045 \mathrm{f}$

\section{References}

1. M. V. Kovalenko, L. Manna, A. Cabot, Z. Hens, D. V. Talapin, C. R. Kagan, V. I. Klimov, A. L. Rogach, P. Reiss, D. J. Milliron, P. Guyot-Sionnnest, G. Konstantatos, W. J. Parak, T. Hyeon, B. A. Korgel, C. B. Murray and W. Heiss, ACS Nano, 2015, 9, 1012-1057; D. V. Talapin, J.S. Lee, M. V. Kovalenko and E. V. Shevchenko, Chem. Rev., 2009, 110, 389-458.

2. Z. Nie, A. Petukhova and E. Kumacheva, Nat. Nanotechnol., 2010, 5, 15-25.

3. G. Decher, Science, 1997, 277, 1232-1237. 
4. E. Seyrek and G. Decher, in Polymer Science: A Comprehensive Reference, ed. K. Matyjaszewski and M. Möller, Elsevier, Amsterdam, 2012, vol. 7, pp. 159-185.

5. S. Srivastava and N. A. Kotov, Acc. Chem. Res., 2008, 41, 1831-1841; Z. Tang, Y. Wang, P. Podsiadlo and N. A. Kotov, Adv. Mater., 2006, 18, 3203-3224.

6. B. P. Pichon, P. Louet, O. Felix, M. Drillon, S. Begin-Colin and G. Decher, Chem. Mater., 2011, 23, 3668-3675.

7. S. Vial, I. Pastoriza-Santos, J. Pérez-Juste and L. M. Liz- Marzán, Langmuir, 2007, 23, 46064611.

8. Y. Wang, Z. Tang, P. Podsiadlo, Y. Elkasabi, J. Lahann and N. A. Kotov, Adv. Mater., 2006, $18,518-522$.

9. S. Lal, J. H. Hafner, N. J. Halas, S. Link and P. Nordlander, Acc. Chem. Res., 2012, 45, 18871895.

10. Y. Xia, P. Yang, Y. Sun, Y. Wu, B. Mayers, B. Gates, Y. Yin, F. Kim and H. Yan, Adv. Mater., 2003, 15, 353-389.

11. Z. Liu, J. Xu, D. Chen and G. Shen, Chem. Soc. Rev., 2015, 44, 161-192; N. P. Dasgupta, J. Sun, C. Liu, S. Brittman, S. C. Andrews, J. Lim, H. Gao, R. Yan and P. Yang, Adv. Mater., 2014, 26, 2137-2184.

12. Y. Sun, Nanoscale, 2010, 2, 1626-1642; M. Rycenga, C. M. Cobley, J. Zeng, W. Li, C. H. Moran, Q. Zhang, D. Qin and Y. Xia, Chem. Rev., 2011, 111, 3669-3712.

13. L. Daniel, G. Gaël, M. Céline, C. Caroline, B. Daniel and S. Jean-Pierre, Nanotechnology, 2013, 24, 452001; M. Layani, A. Kamyshny and S. Magdassi, Nanoscale, 2014, 6, 55815591; W. He and C. Ye, J. Mater. Sci. Technol., 2015, 31, 581-588; S. Ye, A. R. Rathmell, Z. Chen, I. E. Stewart and B. J. Wiley, Adv. Mater., 2014, 26, 6670-6687.

14. X. Xiong, C.-L. Zou, X.-F. Ren, A.-P. Liu, Y.-X. Ye, F.-W. Sun and G.-C. Guo, Laser Photonics Rev., 2013, 7, 901-919; X. Guo, Y. Ma, Y. Wang and L. Tong, Laser Photonics Rev., 2013, 7, 855-881; Y. Huang, Y. Fang, Z. Zhang, L. Zhu and M. Sun, Light: Sci. Appl., 2014, 3, e199.

15. T. Shegai, V. D. Miljković, K. Bao, H. Xu, P. Nordlander, P. Johansson and M. Käll, Nano Lett., 2011, 11, 706-711.

16. S. J. Lee, J. M. Baik and M. Moskovits, Nano Lett., 2008, 8, 3244-3247; R. F. Aroca, P. J. G. Goulet, D. S. dos Santos, R. A. Alvarez-Puebla and O. N. Oliveira, Anal. Chem., 2004, 77, 378-382.

17. J.-W. Liu, J.-L. Wang, W.-R. Huang, L. Yu, X.-F. Ren, W.-C. Wen and S.-H. Yu, Sci. Rep., 2012, 2, 987.

18. J.-W. Liu, H.-W. Liang and S.-H. Yu, Chem. Rev., 2012, 112, 4770-4799; M. Kwiat, S. Cohen, A. Pevzner and F. Patolsky, Nano Today, 2013, 8, 677-694; Y.-Z. Long, M. Yu, B. Sun, C.-Z. Gu and Z. Fan, Chem. Soc. Rev., 2012, 41, 4560- 4580.

19. A. R. Tao, J. Huang and P. Yang, Acc. Chem. Res., 2008, 41, 1662-1673; V. Krishnan, Y. Kasuya, Q. Ji, M. Sathish, L. K. Shrestha, S. Ishihara, K. Minami, H. Morita, T. Yamazaki, N. Hanagata, K. i. Miyazawa, S. Acharya, W. Nakanishi, J. P. Hill and K. Ariga, ACS Appl. Mater. Interfaces, 2015, 7, 15667-15673.

20. M. Chen, I. Y. Phang, M. R. Lee, J. K. W. Yang and X. Y. Ling, Langmuir, 2013, 29, 70617069.

21. G. Yu, A. Cao and C. M. Lieber, Nat. Nanotechnol., 2007, 2, 372-377.

22. T. Ackermann, R. Neuhaus and S. Roth, Sci. Rep., 2016, 6, 34289.

23. B. Park, I.-G. Bae and Y. H. Huh, Sci. Rep., 2016, 6, 19485.

24. Y. Fang, K. Ding, Z. Wu, H. Chen, W. Li, S. Zhao, Y. Zhang, L. Wang, J. Zhou and B. Hu, ACS Nano, 2016, 10, 10023-10030. 
25. S. Kang, T. Kim, S. Cho, Y. Lee, A. Choe, B. Walker, S.-J. Ko, J. Y. Kim and H. Ko, Nano Lett., 2015, 15, 7933-7942.

26. C. M. Hangarter and N. V. Myung, Chem. Mater., 2005, 17, 1320-1324; S. Raychaudhuri, S. A. Dayeh, D. Wang and E. T. Yu, Nano Lett., 2009, 9, 2260-2266.

27. Y. Huang, X. Duan, Q. Wei and C. M. Lieber, Science, 2001, 291, 630-633.

28. K. M. O. Håkansson, A. B. Fall, F. Lundell, S. Yu, C. Krywka, S. V. Roth, G. Santoro, M. Kvick, L. Prahl Wittberg, L. Wågberg and L. D. Söderberg, Nat. Commun., 2014, 5, 4018.

29. O. Assad, A. M. Leshansky, B. Wang, T. Stelzner, S. Christiansen and H. Haick, ACS Nano, 2012, 6, 4702-4712.

30. R. Blell, X. Lin, T. Lindstrom, M. Ankerfors, M. Pauly, O. Felix and G. Decher, ACS Nano, 2016, DOI: 10.1021/acsnano.6b04191.

31. S. Sekar, V. Lemaire, H. Hu, G. Decher and M. Pauly, Faraday Discuss., 2016, 191, 373389.

32. Y.-K. Kim, P. S. Kang, D.-I. Kim, G. Shin, G. T. Kim and J. S. Ha, Small, 2009, 5, 727-734.

33. B. S. Shim and N. A. Kotov, Langmuir, 2005, 21, 9381-9385.

34. C. Yang, H. Gu, W. Lin, M. M. Yuen, C. P. Wong, M. Xiong and B. Gao, Adv. Mater., 2011, 23, 3052-3056.

35. R. Rezakhaniha, A. Agianniotis, J. T. C. Schrauwen, A. Griffa, D. Sage, C. V. C. Bouten, F. N. Vosse, M. Unser and N. Stergiopulos, Biomech. Model. Mechanobiol., 2012, 11, 461-473.

36. C. A. Schneider, W. S. Rasband and K. W. Eliceiri, Nat. Methods, 2012, 9, 671-675; M. D. Abramoff, P. J. Magalhaes and S. J. Ram, Biophotonics Int., 2004, 11, 36-42.

37. E. Prodan, C. Radloff, N. J. Halas and P. Nordlander, Science, 2003, 302, 419-422; P. Nordlander, C. Oubre, E. Prodan, K. Li and M. I. Stockman, Nano Lett., 2004, 4, 899-903; N. J. Halas, S. Lal, W.-S. Chang, S. Link and P. Nordlander, Chem. Rev., 2011, 111, 39133961.

38. J. P. Kottmann, O. J. F. Martin, D. R. Smith and S. Schultz, Phys. Rev. B: Condens. Matter, 2001, 64, 235402.

39. M. S. Goh, Y. H. Lee, S. Pedireddy, I. Y. Phang, W. W. Tjiu, J. M. R. Tan and X. Y. Ling, Langmuir, 2012, 28, 14441-14449; Y. Gao, P. Jiang, L. Song, L. Liu, X. Yan, Z. Zhou, D. Liu, J. Wang, H. Yuan, Z. Zhang, X. Zhao, X. Dou, W. Zhou, G. Wang and S. Xie, J. Phys. D: Appl. Phys., 2005, 38, 1061.

40. F.-X. Xiao, M. Pagliaro, Y.-J. Xu and B. Liu, Chem. Soc. Rev., 2016, 45, 3088-3121; J. J. Richardson, M. Björnmalm and F. Caruso, Science, 2015, 348, aaa2491. 